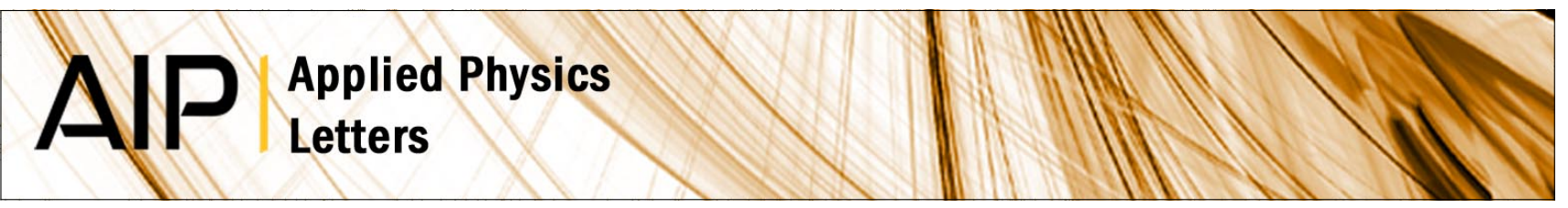

\title{
Observation of spin dependent photocoductivity in InSb quantum well nanowires
}

Juerong Li, A. M. Gilbertson, K. L. Litvinenko, L. F. Cohen, and S. K. Clowes

Citation: Appl. Phys. Lett. 101, 152407 (2012); doi: 10.1063/1.4760223

View online: http://dx.doi.org/10.1063/1.4760223

View Table of Contents: http://apl.aip.org/resource/1/APPLAB/v101/i15

Published by the American Institute of Physics.

\section{Related Articles}

Extrinsic charge traps in disordered organic materials

J. Appl. Phys. 112, 073715 (2012)

Theory of photoferroelectric response in $\mathrm{SmC}^{*}$ liquids

J. Chem. Phys. 137, 144902 (2012)

Electronically active defects in the $\mathrm{Cu} 2 \mathrm{ZnSn}(\mathrm{Se}, \mathrm{S}) 4$ alloys as revealed by transient photocapacitance spectroscopy

Appl. Phys. Lett. 101, 142106 (2012)

Determination of electron and hole lifetimes of rutile and anatase $\mathrm{TiO} 2$ single crystals

Appl. Phys. Lett. 101, 133907 (2012)

Negative and positive photoconductivity modulated by light wavelengths in carbon nanotube film

Appl. Phys. Lett. 101, 123117 (2012)

\section{Additional information on Appl. Phys. Lett.}

Journal Homepage: http://apl.aip.org/

Journal Information: http://apl.aip.org/about/about_the_journal

Top downloads: http://apl.aip.org/features/most_downloaded

Information for Authors: http://apl.aip.org/authors

\section{ADVERTISEMENT}

AMERICAN

PHYSICAL

SOCIETY'S

OPEN ACCESS

JOURNAL

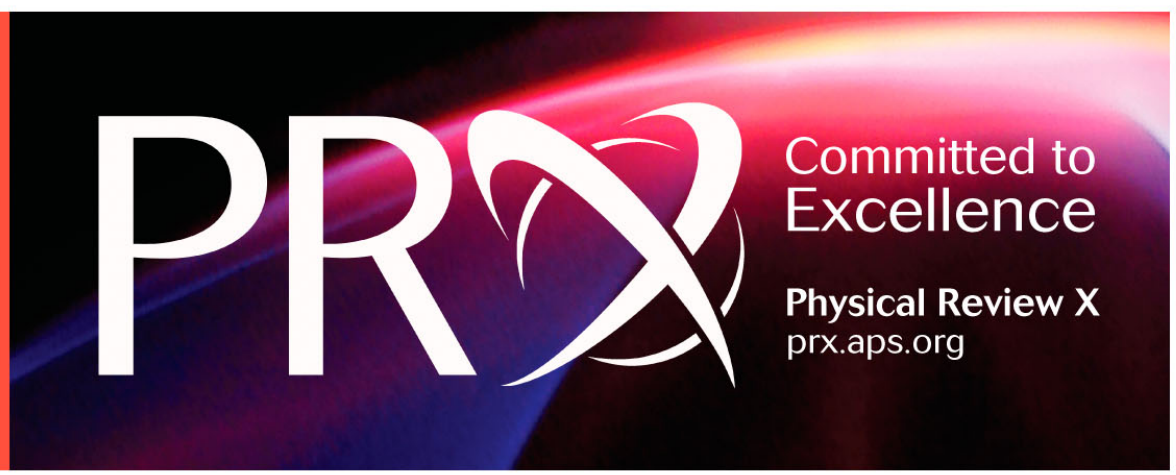




\title{
Observation of spin dependent photocoductivity in InSb quantum well nanowires
}

\author{
Juerong Li, ${ }^{1}$ A. M. Gilbertson, ${ }^{2}$ K. L. Litvinenko, ${ }^{1}$ L. F. Cohen, ${ }^{2}$ and S. K. Clowes ${ }^{1, a)}$ \\ ${ }^{1}$ Advanced Technology Institute and SEPNet, University of Surrey, Guildford GU2 7XH, United Kingdom \\ ${ }^{2}$ Blackett Laboratory, Imperial College London, Prince Consort Rd, London SW7 2BZ, United Kingdom
}

(Received 16 July 2012; accepted 4 October 2012; published online 12 October 2012)

\begin{abstract}
We report on the electrical detection of spin dependent photoconductivity in $500 \mathrm{~nm}$ wide $\mathrm{InSb}$ quantum well nanowires using the optical orientation of electron spins. By applying weak magnetic fields $(\approx 200 \mathrm{mT})$, we observe a spin filtering effect of classical origin caused by spin dependent back scattering of electrons from the sidewalls. Spin dependent features in the longitudinal photovoltage decay with temperature and disappears at characteristic energy $(\approx 50 \mathrm{~K})$ consistent with the theoretical spin splitting and the thermal level broadening. We show that the observed signal is due to the inversion asymmetry of the quantum well, with an additional Zeeman contribution. (C) 2012 American Institute of Physics. [http://dx.doi.org/10.1063/1.4760223]
\end{abstract}

The preparation and manipulation of spin-polarized carriers in semiconductors have been the subject of intense interest for the past decade. Pure spin currents and spinpolarized charge currents induced by electric current alone have been demonstrated in finite three dimensional and two dimensional systems with spin-orbit (SO) interaction by the spin Hall effect ${ }^{1}$ and the current-induced spin polarization (CISP), ${ }^{2,3}$ respectively. The latter is a manifestation of the internal effective magnetic field generated by the $k$-linear SO splitting and represents the inverse circular-photo galvanic effect. These studies have focused on systems that are geometrically rather wide where bulk properties of the system dominate. Quantum wires are interesting systems to study spin dependent transport because as the dimensions of conducting channels in semiconductor devices become ever smaller, the role of electron-boundary interactions becomes increasingly important. ${ }^{4}$ These interactions are apparent in classical magneto-size effects of quasi-one dimensional (1D) channels (quantum wires) where charge transport between the boundaries is ballistic. ${ }^{4,5}$ Equally, the reduced dimensionality has a remarkable effect on the spin dynamics, e.g., suppression of D'yakonov-Perel spin relaxation and enhanced phase coherence lengths. ${ }^{6}$ In this letter, we report the electrical detection of spin-polarized charge currents in InSb quantum wires with large spin-orbit coupling using the optical spin orientation technique described in Ref. 7.

In general, as the width ( $w$ ) of a channel is decreased, its resistivity will increase due to carrier back-scattering from boundary imperfections that can be considered at least partially diffuse (the exception is the case where the boundaries are perfectly smooth and scattering is specular). In a perpendicular magnetic field $(B)$, carriers undergo cyclotron motion with a radius given by $R_{c}=\hbar k_{F} / e B$. This increases the degree of interaction with the boundaries, but the effect on the resistivity is less trivial, e.g., in weak magnetic fields such that $B<2 B_{0}=2 \hbar k_{F} / e w$, a nonmonotonic magnetoresistance can manifest. Experimentally, this is characterized

\footnotetext{
${ }^{\text {a) }}$ Author to whom correspondence should be addressed. Electronic mail: s.clowes@surrey.ac.uk.
}

by a peak in the resistivity at $B=B_{1} \approx 0.7 B_{0}$ corresponding to maximum backscattering. ${ }^{8,9}$ For $B>2 B_{0}$, skipping orbits (edge states) are formed that suppress back-scattering. In the presence of a SO interaction, the cyclotron radius is dependent on the orientation of the carrier $\operatorname{spin}^{7,10}$ and consequently so too should the value of $B_{1}$. In analogy to a ferromagnetic metal, a spin polarized current will be produced at $B=B_{1}$ due to the asymmetry in the resistivity of the spin dependent current channels, which in the case of a semiconductor nanowire is governed by the strength of the SO interaction and by the dominant mechanism for boundary scattering. The critical spin dependent cyclotron radius can be obtained using

$$
R_{\sigma}=\frac{\hbar k_{\sigma}}{e B}
$$

where $k_{\sigma}$ is a spin dependent momentum, given by

$$
k_{\sigma}=\sqrt{k_{F}^{2}-\frac{m^{*} \sigma|\Omega|}{\hbar}}, \quad \sigma=\frac{\mathbf{S} . \Omega}{|\mathbf{S}||\Omega|},
$$

where $k_{F}$ is the Fermi momentum, $\sigma$ is the projection of spin along precession vector $\Omega$ (taking values from -1 to +1 ), $m^{*}$ is the electron effective mass, and $\mathbf{S}$ is the spin vector. Equation (2) can be derived from Eqs. (2)-(4) in Ref. 7. Note that the usual spin independent radius is obtained when $\sigma=0$.

The sample used is a modulation doped $\mathrm{InSb} / \mathrm{InAlSb}$ quantum well (QW) heterostructure grown by molecularbeam epitaxy (MBE) on semi-insulating GaAs (001). ${ }^{11} \mathrm{~A}$ $30 \mathrm{~nm}$ wide $\mathrm{InSb} \mathrm{QW}$ is buried $50 \mathrm{~nm}$ beneath the surface. Electrons are supplied by a Te $\delta$-layer located $20 \mathrm{~nm}$ above the top of the QW in the $50 \mathrm{~nm} \mathrm{Al}_{0.15} \mathrm{In}_{0.85} \mathrm{Sb}$ cap layer. The sheet carrier concentration and mobility $(\mu)$ of the as grown QW at $2 \mathrm{~K}$ were $n=3.95 \times 10^{15} \mathrm{~m}^{-2}$ and $\mu=19.5 \mathrm{~m}^{2} \mathrm{~V}^{-1} \mathrm{~s}^{-1} .{ }^{9} \mathrm{~A}$ quantum wire with $w \approx 500 \mathrm{~nm}$ was patterned by shallow reactive ion etching in a $\mathrm{CH}_{4} / \mathrm{H}_{2}$ plasma to a depth of $100 \mathrm{~nm}$. A longitudinal voltage lead separation of $8.4 \mu \mathrm{m}$ ensures that transport is diffusive along the channel. The sheet carrier concentration and mobility of the quantum wire were $n=3.77 \times 10^{15} \mathrm{~m}^{-2}$ and $\mu=14.8 \mathrm{~m}^{2} /$ Vs. As the edges of the channel are exposed to atmosphere, traps and surface 
damaging will produce depletion in the two dimensional electron gas near the channel edges. ${ }^{12,13}$ The effective channel width is estimated to be $w_{\text {eff }} \approx 400 \mathrm{~nm} .{ }^{9}$

Measurements were performed in a $7 \mathrm{~T}$ magneto-optical cryostat with $\mathrm{CaF}$ and $\mathrm{ZnSe}$ windows. A $2 \mathrm{~V}$ dc voltage was applied across the Hall bar contacts 1 and 2 [see Fig. 1(a)] and a $1 \mathrm{M} \Omega$ series resistor supplying a dc current of $\approx 2 \mu \mathrm{A}$. A Ti:sapphire fs ultrafast laser operating at a repetition frequency of $250 \mathrm{kHz}$ was tuned to a wavelength of $4 \mu \mathrm{m}$ with a power less than $1 \mathrm{~mW}$ at the sample position and modulated at a frequency of $1 \mathrm{kHz}$. In the experimental arrangement, the light is incident close to the sample normal. In order to excite spin-polarized carriers, a $\mathrm{ZnSe}$ linear polarizer and a photo-elastic modulator (PEM) were used to modulate the helicity of the incident light from left-handed circular polarization $\left(\sigma_{-}\right)$to right-hand circular polarization $\left(\sigma_{+}\right)$at a frequency of $37 \mathrm{kHz}$. This in turn produces a modulation of the preferential spin orientation due to the selection rules of the photo-excited electrons. ${ }^{7}$ Both the longitudinal spin-dependent, $V_{\text {heli }}\left(\propto \sigma_{+}-\sigma_{-}\right.$at $\left.37 \mathrm{kHz}\right)$, and spinindependent, $V_{\text {photo }}(1 \mathrm{kHz})$, photo responses are detected between contacts 3 and 4 by standard lock-in technique. Note that a change of sign in $\sigma$ will cause a $\pi$ phase shift in $V_{\text {heli }}$ signal and will have no effect on $V_{\text {photo }}$. The PEM was therefore removed when $V_{\text {photo }}$ was measured in order to maximize the signal. We note that the photon energy is above the InSb QW subband edge $(\approx 310 \mathrm{meV})$ but it is below the band edge of InAlSb barrier layers. In this way, we eliminate any parallel (photo) conduction from the AlInSb cap and buffer layers (such parallel conduction complicates conventional transport measurements).

In Fig. 1(b), we compare the Shubnikov de Haas (SdH) oscillations from a macroscopic $40 \mu \mathrm{m}$ wide Hall bar of the reported InSb QW sample obtained for the dark resistance $\left(R_{d a r k}\right)$ and the signal under illumination $\left(V_{\text {photo }}\right)$. A $9 \%$ increase in the spin degenerate carrier concentration is observed in $V_{\text {photo }}$ due to the photoexcitation of additional charge carrying electrons. Notably, the resolution of the $\mathrm{SdH}$ oscillations in $V_{\text {photo }}$ is significantly improved, and the distinct

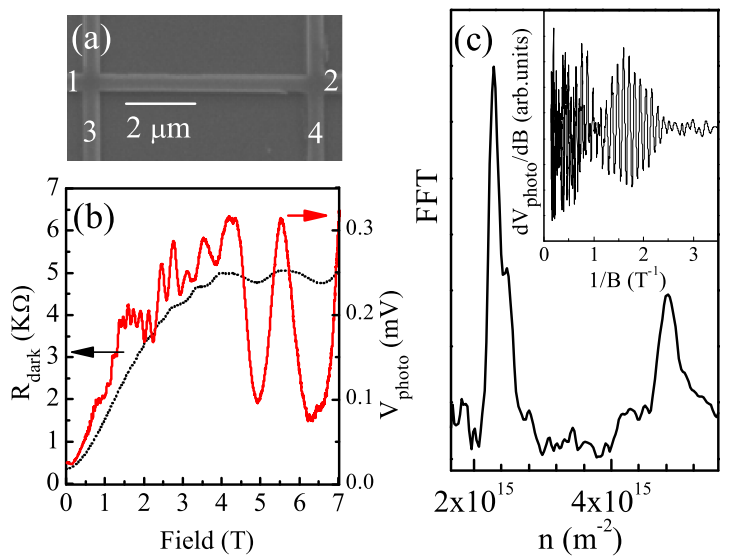

FIG. 1. (a) SEM image of patterned Hall bar structure with wire width of $500 \mathrm{~nm}$ showing contact designation, (b) $R_{\text {dark }}$ and $V_{\text {photo }}$ as a function of $B$ for macroscopic InSb QW Hall bar (40 $\mu$ m wire width), (c) inset: differential $d V_{\text {photo }} / d B$ for macroscopic Hall bar as a function of $1 / B$ showing clear beating and main panel: FFT of $d V_{\text {photo }} / d B$ signal suggesting two spin-split subbands of the main peak $\left(n_{1}=2.06 \times 10^{15} \mathrm{~m}^{-2}\right.$ and $\left.n_{2}=2.23 \times 10^{15} \mathrm{~m}^{-2}\right)$ corresponding to a Rashba coefficient $\alpha=0.11 \mathrm{eVA}$. beating patterns observed in the derivative $d V_{\text {photo }} / d B$, characteristic of SO splitting of the QW sub-band, are to-date the most convincing. This splitting is observed in the Fourier analysis [Fig. 1(c)] from which a Rashba coefficient of $\alpha=0.11 \mathrm{eV} \AA$ is deduced, comparable to that previously measured in InSb QWs and demonstrating the strong SO interaction in this system. ${ }^{11}$ The Dresselhaus effect is not considered here as calculations have shown the Rashba interaction to be dominant in this type of heterostructure. ${ }^{11,14}$

The photoresponse measurements on the nanowire at $10 \mathrm{~K}$ are shown in Fig. 2(a). In $V_{\text {photo }}$, we observe oscillations at $|B|>2 B_{0} \approx 0.5 \mathrm{~T}$ that are attributed to magnetodepopulation of 1D subbands. ${ }^{9,15}$ Features observed for $B<2 B_{0}$ are thus related to boundary scattering. We point out the minima which occur at magnetic fields $\pm 170 \mathrm{mT}$ that we denote $\pm B_{1}$, which are coincident with the observed maxima in electrical resistivity. ${ }^{9}$ This observed inversion of $V_{\text {photo }}$ is due to this differential signal being proportional to the negative term $\left(1 / n_{\text {photo }}-1 / n\right)$, where $n_{\text {photo }}$ and $n$ are the respective carrier concentrations with and without illumination. A non-zero spin-dependent photoresponse $\left(V_{\text {heli }}\right)$ is observed for the entire field range. Moreover, oscillatory behavior is also observed in $V_{\text {heli }}$ for $|B|<0.5 \mathrm{~T}$ that appear to be in anti-phase with respect to positive and negative applied magnetic field (see dashed lines in Fig. 2). Before discussing these effects further, we note that the non-zero $\mathrm{V}_{\text {heli }}$ is itself of interest. Illumination of (001) grown QWs of

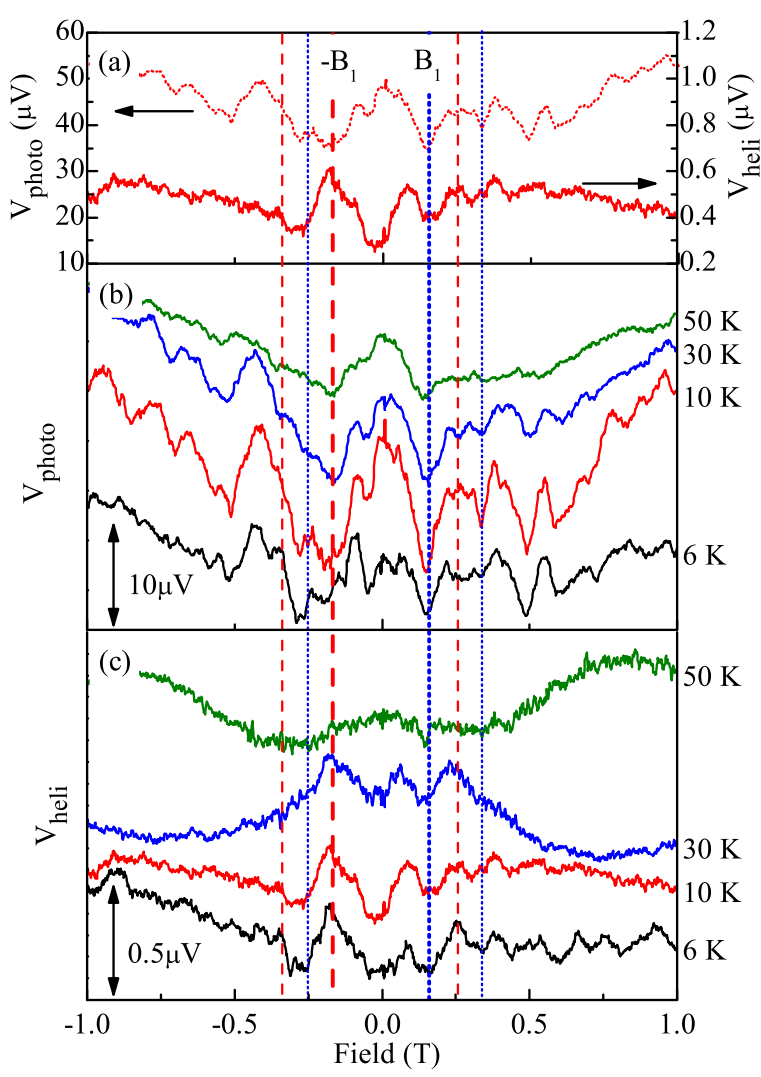

FIG. 2. $V_{\text {photo }}$ and $V_{\text {heli }}$ of the nanoscale Hall bar device measured using $4 \mu \mathrm{m}$ laser. Comparison of $V_{\text {photo }}$ and $V_{\text {heli }}$ at $10 \mathrm{~K}$ (a) and temperature dependence of $V_{\text {photo }}$ (b) and $V_{\text {heli }}$ (c). The two minima in the $V_{\text {photo }}$ at $\pm 170 \mathrm{mT}$ in photoresponse corresponding to $B= \pm B_{1}$ are indicated by dashed lines. Additional guides for the eye are included which indicate minima (blue dotted) and peaks (red dashed) in $V_{\text {heli }}$ at \pm 250 and $\pm 330 \mathrm{mT}$. 
high symmetry under normal incidence light excludes the circular photogalvanic and circular spin-galvanic effects that can lead to helicity dependent spin photo-currents in unbiased samples. Indeed, we do not find any sizable $\mathrm{V}_{\text {heli }}$ response in the nanowire without an applied bias (not shown). The applied electric field $\left(E_{x}\right)$ along the nanowire measured between contacts 3 and 4 is $E_{x} \approx 0.5 \mathrm{mV} / \mu \mathrm{m}$. In this scenario, electrons acquire an average drift momentum $\langle k\rangle$ $=\mu m^{*} E_{x} / \hbar$ along the nanowire, inducing a regular effective precession vector $\left\langle\Omega_{e f f}(k)\right\rangle=2 \alpha\langle k\rangle / \hbar$ perpendicular to the nanowire axis in addition to the fluctuating $\Omega$ that contributes to D'yakonov Perel spin relaxation, where we have assumed only the $k$-linear SO interaction. Optically oriented spins $\left(S_{z}\right)$ may precess about $\left\langle\Omega_{e f f}\right\rangle$ into the QW plane; however, since $\left\langle\Omega_{e f f}\right\rangle$ is always perpendicular to the nanowire axis, the magnetogyrotropic photogalvanic effects recently observed in similar InSb QWs are also not permitted. ${ }^{16}$ The influence of CISP effect can be estimated as $\langle\sigma\rangle \approx \hbar\left\langle\Omega_{\text {eff }}\right\rangle / 2 E_{F}$, where $E_{F}$ is the Fermi energy, from which we obtain $\approx 1 \times 10^{-4}$ with $m^{*}=0.013$ and $E_{F}=72 \mathrm{meV}$. While we do not discount the presence of CISP, this value is rather small and we will not consider the influence of dichroic absorption on $\mathrm{V}_{\text {heli }}$. In the absence of the above helicity dependent spin photo-currents, our experiment probes directly the asymmetry of photoconductivity of the spin up and spin down carriers that results from the SO splitting. The features at $\mathrm{B}_{1}$ observed in $\mathrm{V}_{\text {heli }}$ can then be attributed to a spin-filtering effect that results from the backscattering of spins with slightly different cyclotron radii as in Eq. (1). This is a classical magneto-size effect that alters the spin-dependent photoconductivities and is detected in the sensitive PEM technique.

The temperature dependences of $V_{\text {photo }}$ and $V_{\text {heli }}$ up to $50 \mathrm{~K}$ are shown in Figs. 2(b) and 2(c), respectively. As the temperature increases, reproducible fine structure in $V_{\text {photo }}$ disappears except for the dominant minima at $B= \pm B_{1}$. The signal becomes helicity independent by $50 \mathrm{~K}$. At this temperature, carriers have thermal energy of $\approx 4.3 \mathrm{meV}$. This is roughly consistent with the magnitude of the Rashba SO spin-splitting $\Delta=2 \alpha k_{F} \approx 3 \mathrm{meV}$ deduced from Fig. 1 with $\alpha=0.1 \mathrm{eV} \AA$ and $k_{F}=1.57 \times 10^{8} \mathrm{~m}^{-1}$. We also can exclude the Zeeman effect as the associated spin splitting at $170 \mathrm{mT}$ is approximately $0.25 \mathrm{meV}$. We therefore attribute the disappearance of the helicity dependence with temperature to a Rashba mediated spin-dependent transport ${ }^{7}$ which is related to electron spin orientation.

We now return to the observed anti-phase oscillatory structure in $\mathrm{V}_{\text {heli }}(B)$ which we show can be attributed to the experimental arrangement. If the $V_{\text {heli }}$ signal at $B= \pm B_{1}$ in Fig. 2(c) were purely a feature of the Rashba interaction (or even Dresselhaus), one would expect it to be symmetric in $B$ since the orientation of $\Omega$ is only dependent on the direction of $k$ and independent of $B$. In the absence of SO interaction, $\Omega$ acquires a $z$-component proportional to $B$ due to the Zeeman splitting but is contrary to the elevated temperatures to which the features exist as discussed above. The observed asymmetry can be explained by considering the interaction of the Rashba and Zeeman effects when the injected spin orientation is close to the sample normal. As illustrated in Fig. 3(a), at $B=0$, the precession vector $\Omega$ is in the plane of the QW and a non-zero $\sigma$ is only produced when the light is

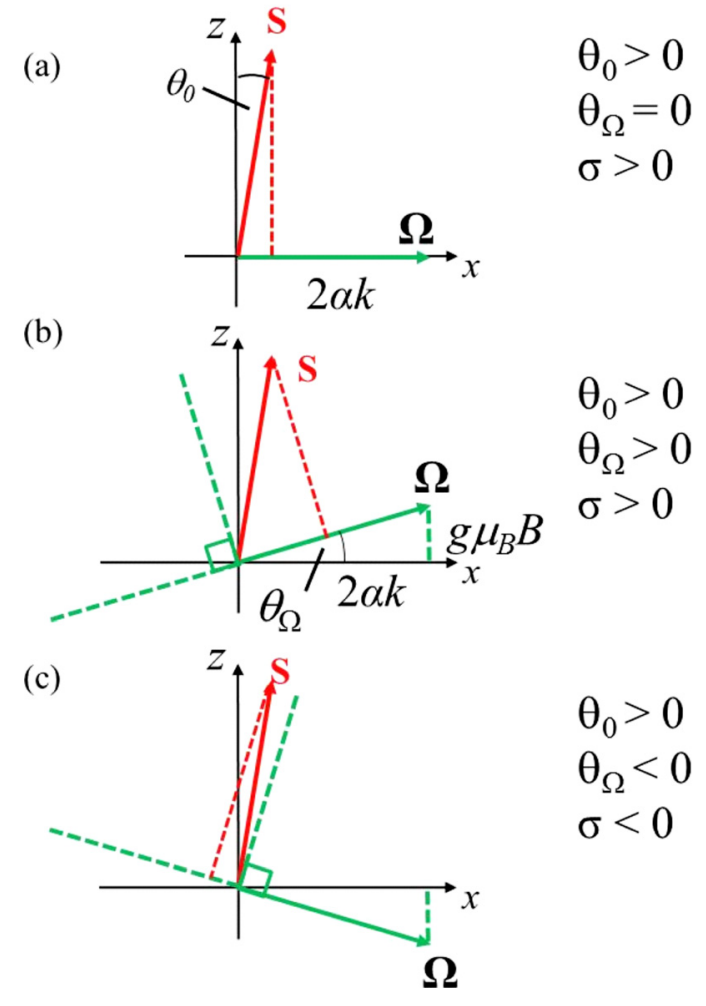

FIG. 3. An illustration showing the sign change of the spins as a function of $B$ field with the electron momentum in the $y$ direction. (a) When $B=0$, there is finite projection of $\mathbf{S}$ along $\Omega(\sigma)$ when $\theta_{0} \neq 0$, (b) $B>0$ the spin projection $\sigma$ is increased, and (c) with sufficient negative $B$ field, $\sigma$ can reverse sign.

incident at a angle $\left(\theta_{0}\right)$ off the normal. In a non-zero magnetic field, the Zeeman splitting lifts $\Omega$ out of $x-y$ plane by an angle $\theta_{\Omega}$ which is given by

$$
\theta_{\Omega}=\tan ^{-1}\left(\frac{g^{*} \mu_{B} B}{2 \alpha k_{F}}\right)
$$

where $g^{*}$ is the effective $g$-factor and $\mu_{B}$ is the Bohr magneton. As shown in Fig. 3(b), when $B>0$ and $\left(\theta_{\Omega}+\theta_{0}\right)>0$, the value of $\sigma$ will be positive and increase with $B$. However, in the case shown in Fig. 3(c) where $B<0$ and $\left(\theta_{0}+\theta_{\Omega}\right)<0$, the value of $\sigma$ will undergo a sign reversal. This will cause a $\pi$ phase shift in the modulation of the spin orientation produced by the PEM, resulting in the asymmetric field response observed in Fig. 2. The condition that $\left(\theta_{\Omega}+\theta_{0}\right)$ and $\sigma$ changes sign for a reversal of $B$ requires a small incident angle $\theta_{0}$ or more specifically the condition $\theta_{\Omega}(B)>\theta_{0}$. For values appropriate for the study $\left(B=B_{1} \approx 170 \mathrm{mT}, \alpha=0.1 \mathrm{eV} \AA, g=30\right.$, $k_{F}=1.57 \times 10^{8} \mathrm{~m}^{-1}$ ), the angle $\theta_{0}$ must be less than approximately $5^{\circ}$ for the Zeeman effect to produce a sign change of $\sigma$. This is indeed the case in our experimental setup where the incident light is close to the sample normal.

In summary, we have observed spin-dependent photoconductivity and classical spin-filtering in InSb nanowire devices using optically orientated spin polarized carriers. The observed temperature dependence is consistent with the helicity dependent response originating from the presence of a SO interaction (Rashba or Dresselhaus). Spin dependent transport of this kind has the potential to act as a spin polarized detector (as in this study) or a spin-filter of unpolarized 
currents. It also demonstrates that for spintronic devices using high SO coupled materials, it is necessary to consider device dimensions and the influence of sidewall scattering.

We would like to thank B. N. Murdin for useful discussions, and J.L. and S.K.C. gratefully acknowledge the support by EPSRC-UK under Grant No. EP/E055583-1 and A.M.G. gratefully acknowledges the support by EPSRC-UK under Grant No. EP/E016243/1.

${ }^{1}$ Y. K. Kato, R. C. Myers, A. C. Gossard, and D. D. Awschalom, Science 306, 1910 (2004).

${ }^{2}$ A. Y. Silov, P. A. Blajnov, J. H. Wolter, R. Hey, K. H. Ploog, and N. S. Averkiev, Appl. Phys. Lett. 85, 5929 (2004).

${ }^{3}$ S. Ganichev, M. Trushin, and J. Schliemann, "Spin polarization by current," in Handbook of Spin Transport and Magnetism (Chapman and Hall/CRC, 2011) pp. 487-495.

${ }^{4}$ C. W. J. Beenakker and H. Vanhouten, Solid State Phys. 44, 1 (1991).

${ }^{5}$ M. Konig, S. Wiedmann, C. Brune, A. Roth, H. Buhmann, L. W. Molenkamp, X. L. Qi, and S. C. Zhang, Science 318, 766 (2007).
${ }^{6}$ A. A. Kiselev and K. W. Kim, Phys. Rev. B 61, 13115 (2000).

${ }^{7}$ J. Li, A. M. Gilbertson, K. L. Litvinenko, L. F. Cohen, and S. K. Clowes, Phys. Rev. B 85, 045431 (2012).

${ }^{8}$ H. Akera and T. Ando, Phys. Rev. B 43, 11676 (1991).

${ }^{9}$ A. M. Gilbertson, M. Fearn, A. Kormnyos, D. E. Read, C. J. Lambert, M. T. Emeny, T. Ashley, S. A. Solin, and L. F. Cohen, Phys. Rev. B 83, 075304 (2011).

${ }^{10}$ L. P. Rokhinson, V. Larkina, Y. B. Lyanda-Geller, L. N. Pfeiffer, and K. W. West, Phys. Rev. Lett. 93, 146601 (2004).

${ }^{11}$ A. M. Gilbertson, W. R. Branford, M. Fearn, L. Buckle, P. D. Buckle, T. Ashley, and L. F. Cohen, Phys. Rev. B 79, 235333 (2009).

${ }^{12}$ T. J. Thornton, Rep. Prog. Phys. 58, 311 (1995).

${ }^{13}$ H. Van houten, B. J. Vanwees, M. G. J. Heijman, and J. P. Andre, Appl. Phys. Lett. 49, 1781 (1986).

${ }^{14}$ M. A. Leontiadou, K. L. Litvinenko, A. M. Gilbertson, C. R. Pidgeon, W. R. Branford, L. F. Cohen, M. Fearn, T. Ashley, M. T. Emeny, B. N. Murdin, and S. K. Clowes, J. Phys. Condens. Matter 23, 035801 (2011).

${ }^{15}$ K.-F. Berggren, G. Roos, and H. van Houten, Phys. Rev. B 37, 10118 (1988).

${ }^{16}$ S. Stachel, P. Olbrich, C. Zoth, U. Hagner, T. Stangl, C. Karl, P. Lutz, V. V. Bel'kov, S. K. Clowes, T. Ashley, A. M. Gilbertson, and S. D. Ganichev, Phys. Rev. B 85, 045305 (2012). 